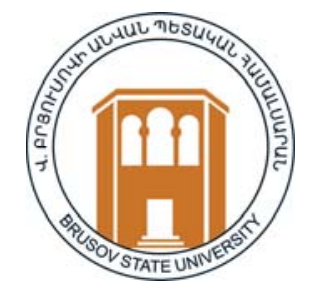

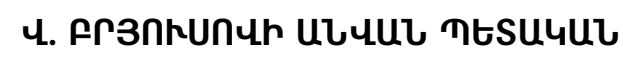
CUUULUURUL

ГОСУДАРСТВЕННЫЙ УНИВЕРСИТЕТ

ИМЕНИ В. БРЮСОВА

BRUSOV STATE UNIVERSITY

คulคtr

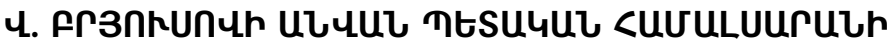
ВЕСТНИК ГОСУДАРСТВЕННОГО УНИВЕРСИТЕТА ИМЕНИ

B. БРЮСОВА

BULLETIN OF BRUSOV STATE UNIVERSITY

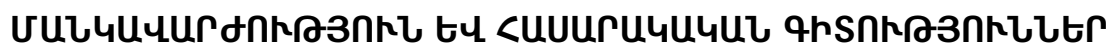

ПЕДАГОГИКА И СОЦИАЛЬНЫЕ НАУКИ

PEDAGOGY AND SOCIAL SCIENCES

2(55)

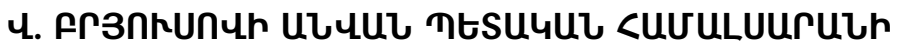

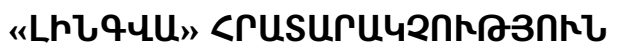

เГヒบuし - 2021 
CS? 378.147:811.111

DOI: 10.51307/182931072015233260/21.2-117

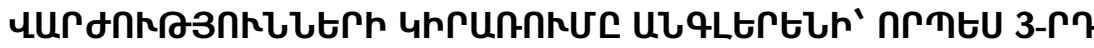

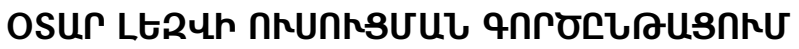

\section{Uhruunrz quRursul}

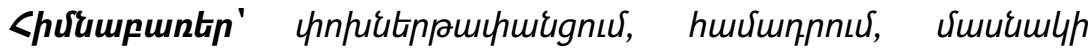

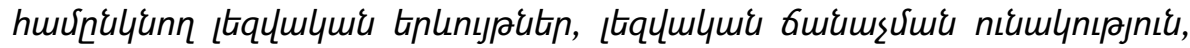

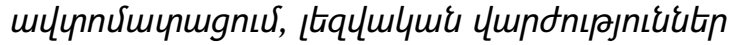

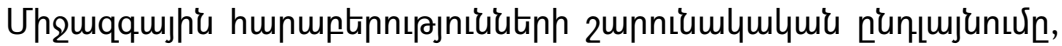

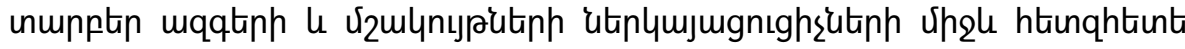

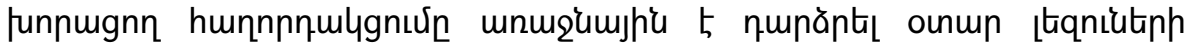

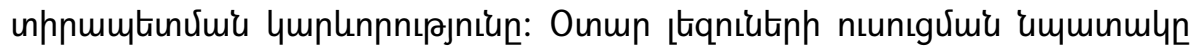

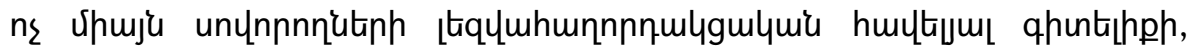

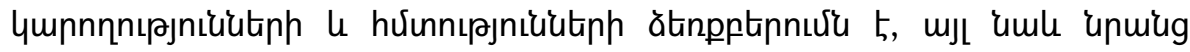

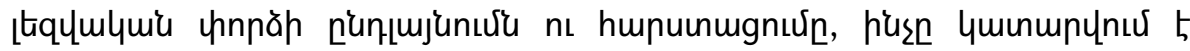

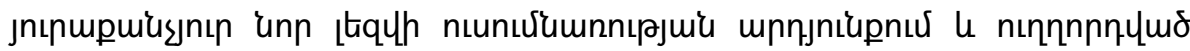

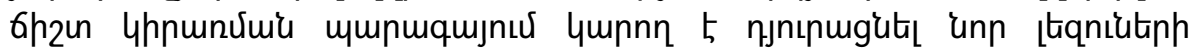

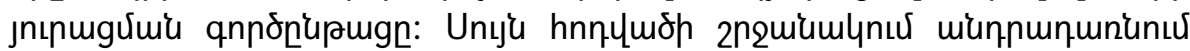

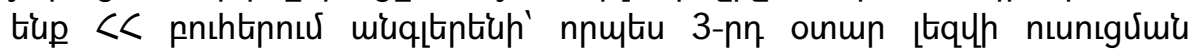

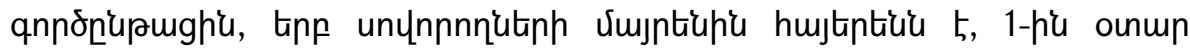

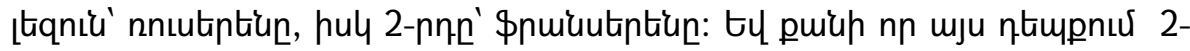

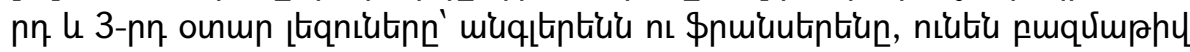

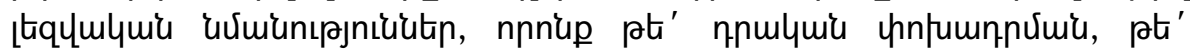

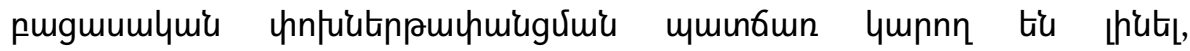

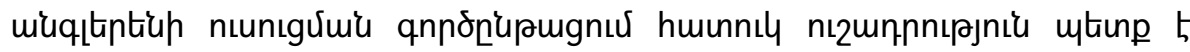

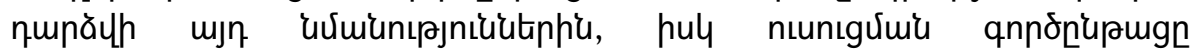

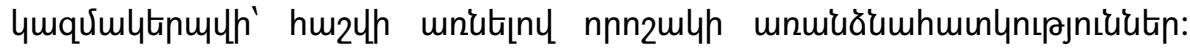

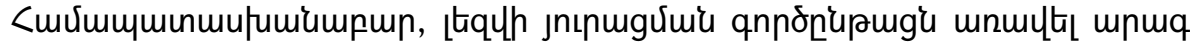

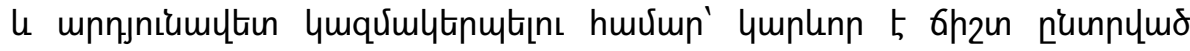

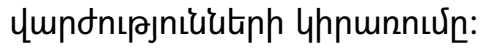

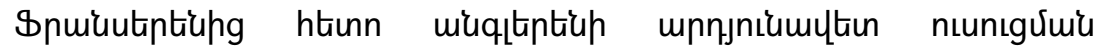

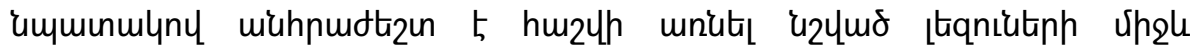

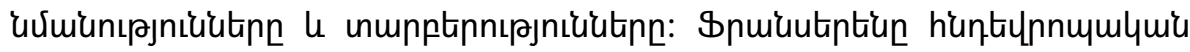

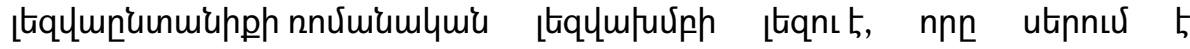




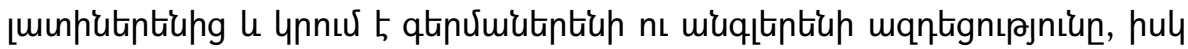

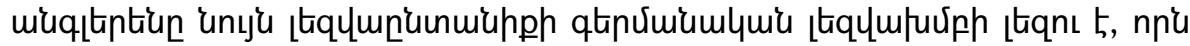

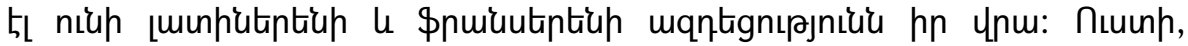

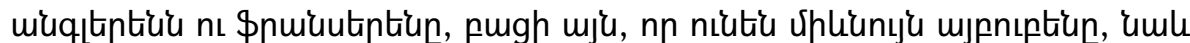

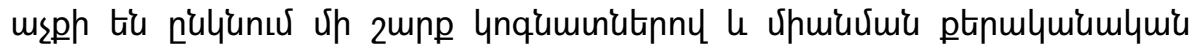

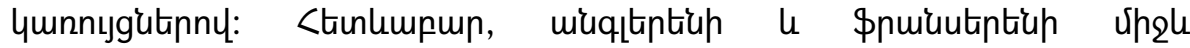

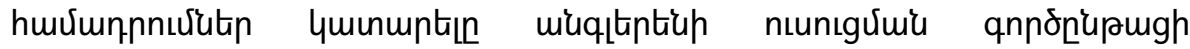

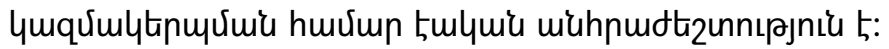

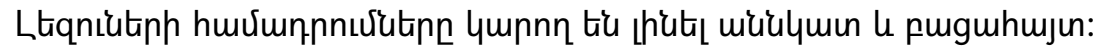

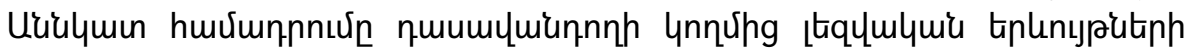

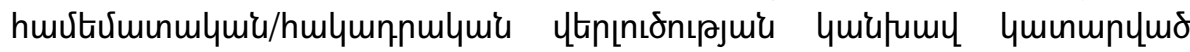

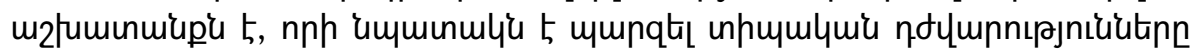

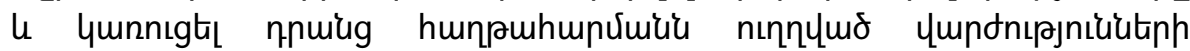

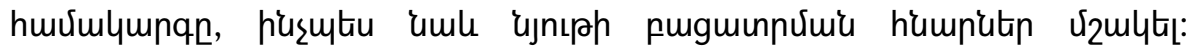

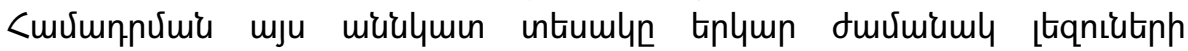

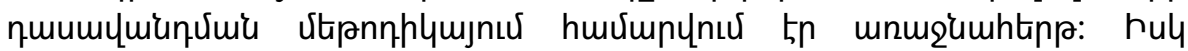

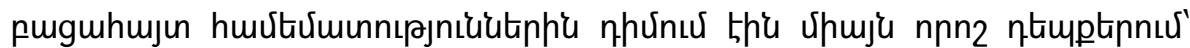

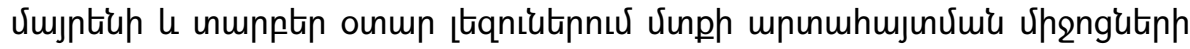

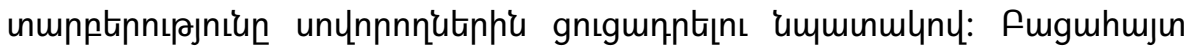

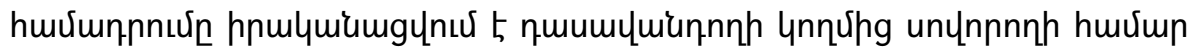

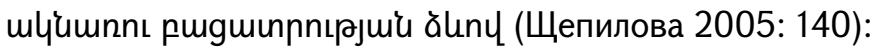

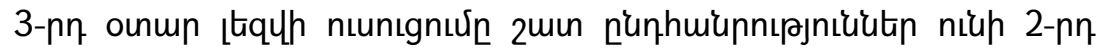

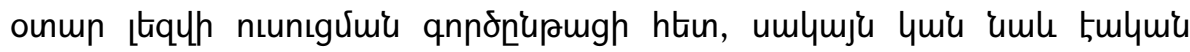

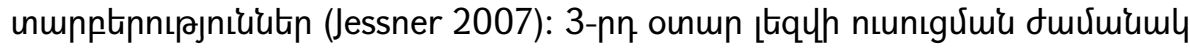

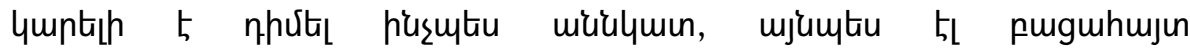

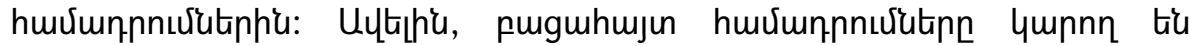

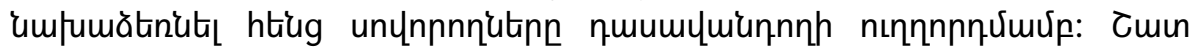

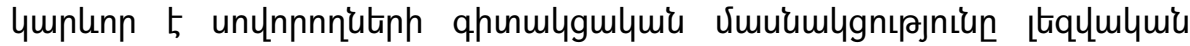

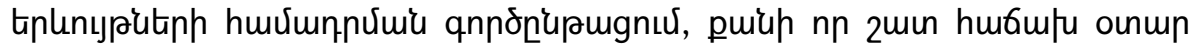

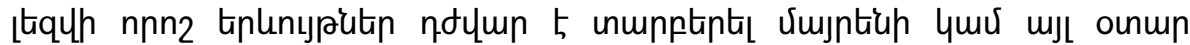

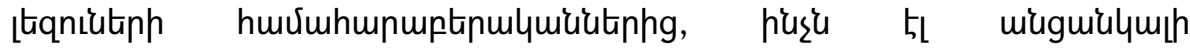

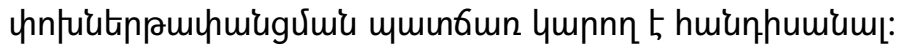

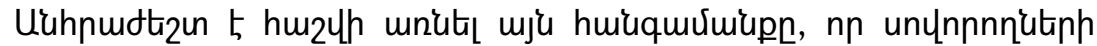

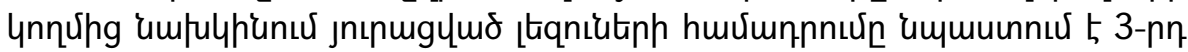

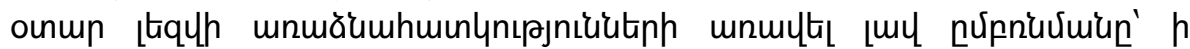

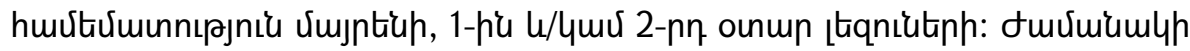

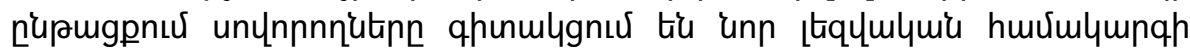

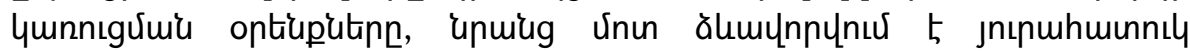

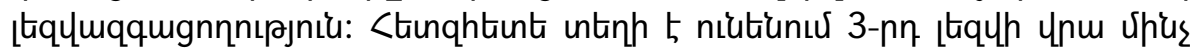




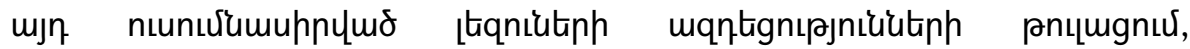

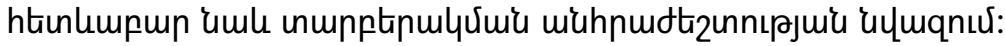

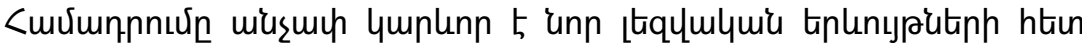

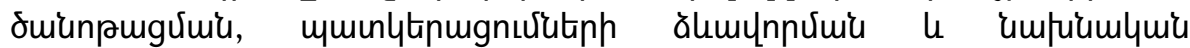

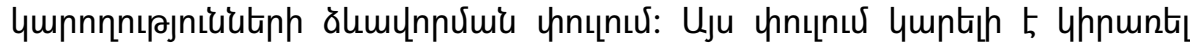

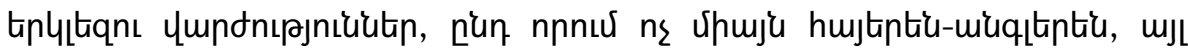

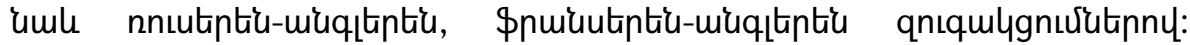

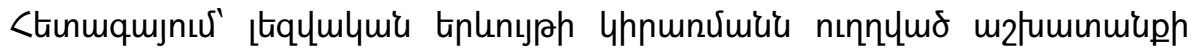

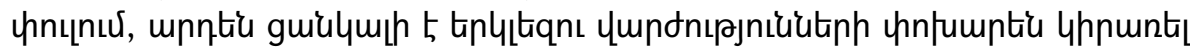

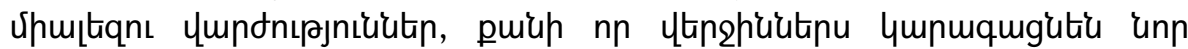

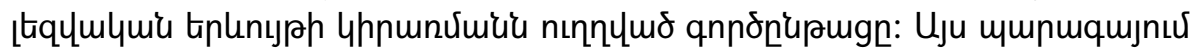

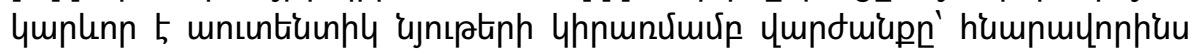
hnmlumir hunnnnulygulquiu úh

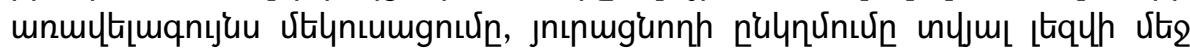

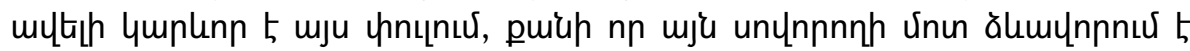

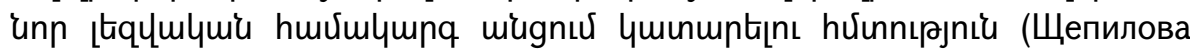
2005: 141):

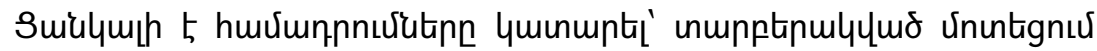

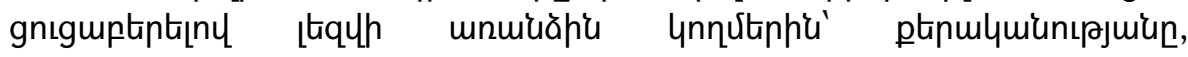

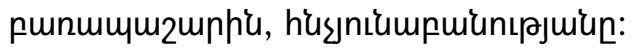

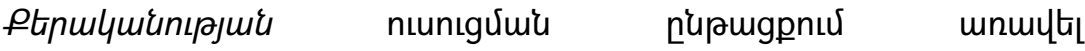

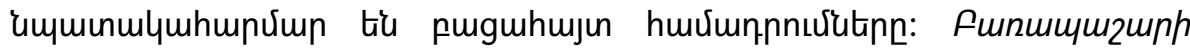

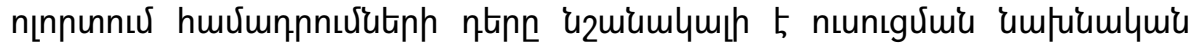

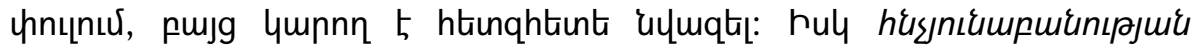

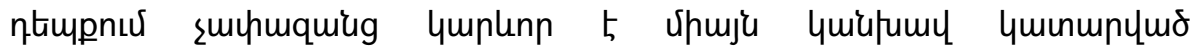

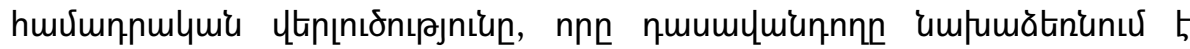

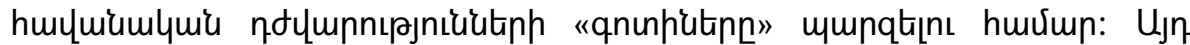

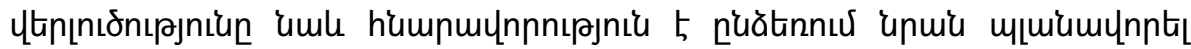

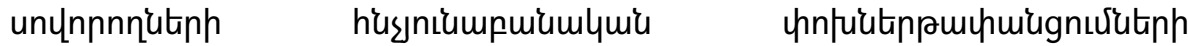

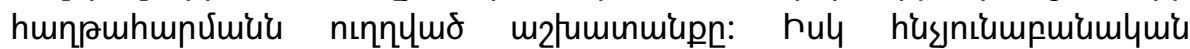

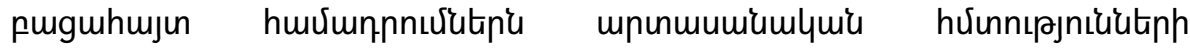

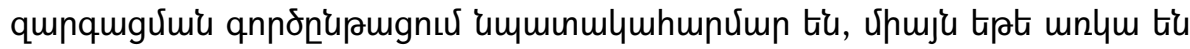

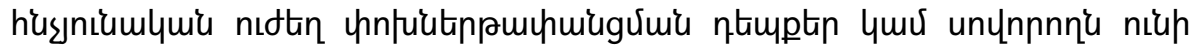

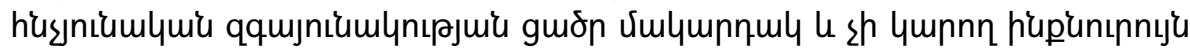

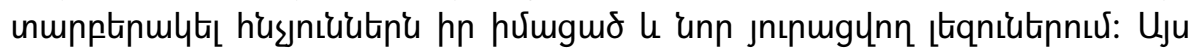

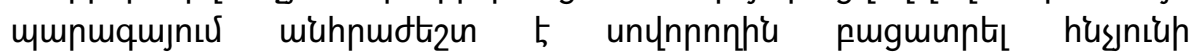

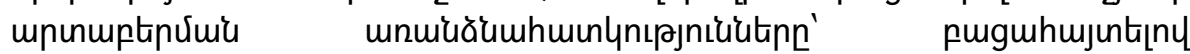

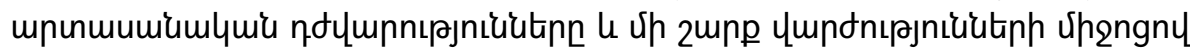

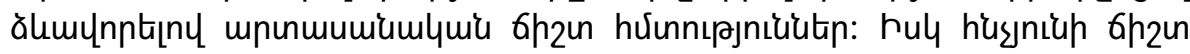




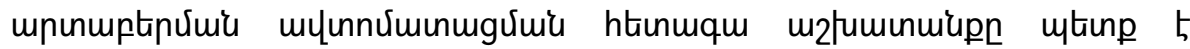

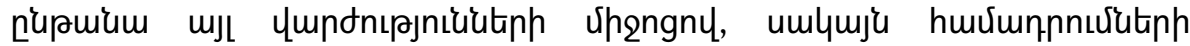
pugunúuर्up (Щепилова 2005: 141):

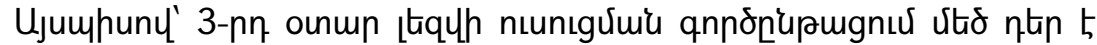

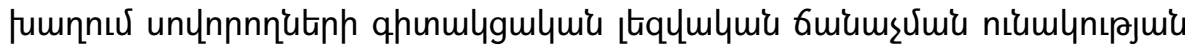

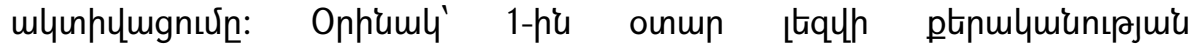

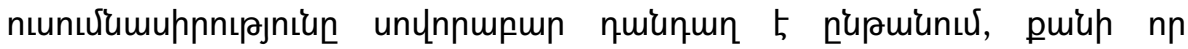

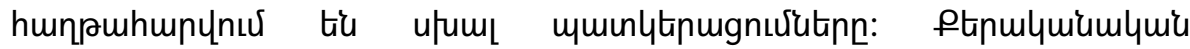

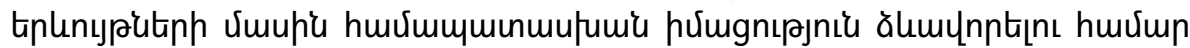

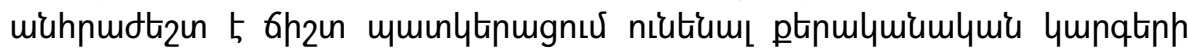

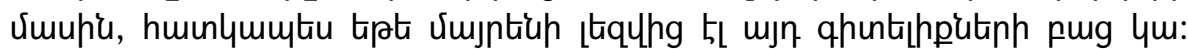

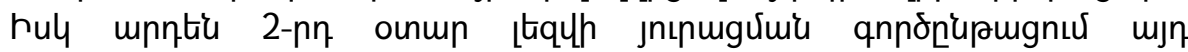

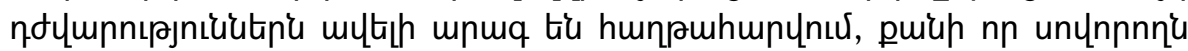

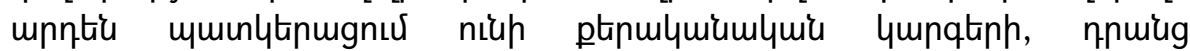

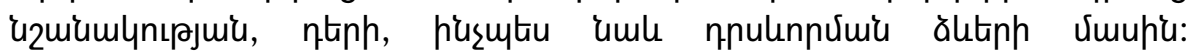
(Щепилова 2005: 135-137):

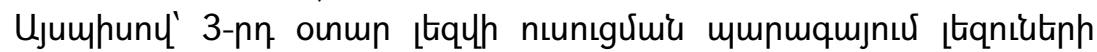

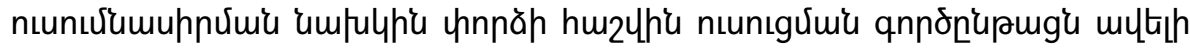

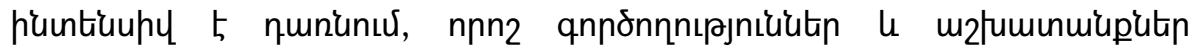

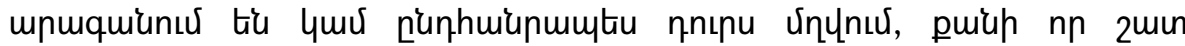

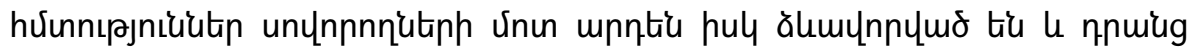

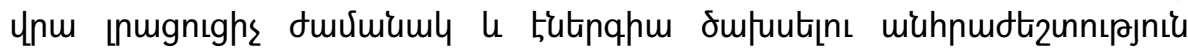

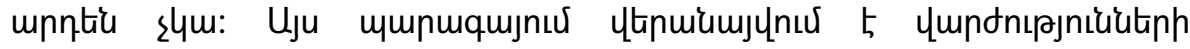

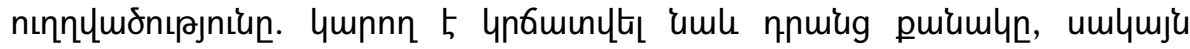

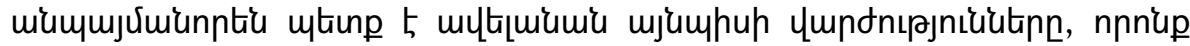

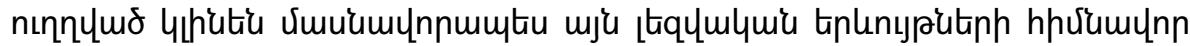

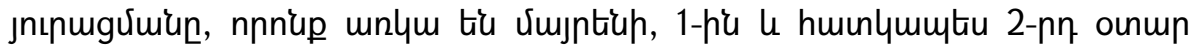

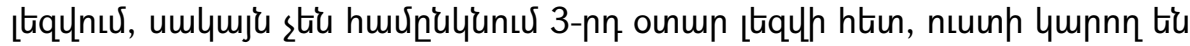

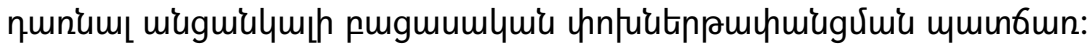

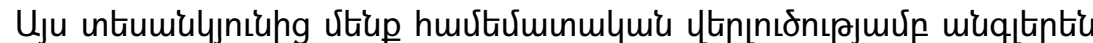

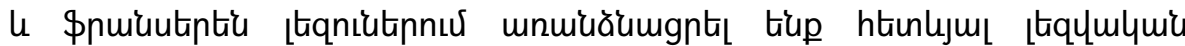

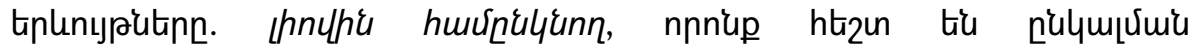

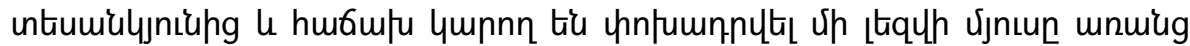

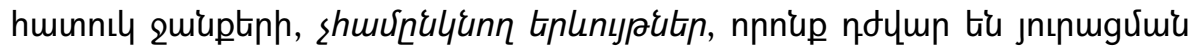

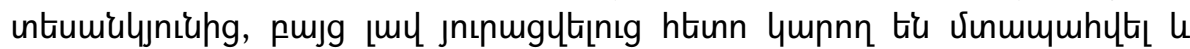

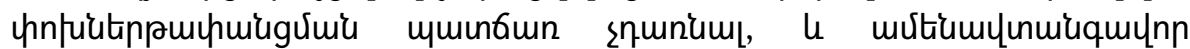

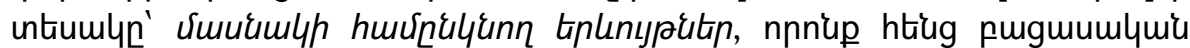

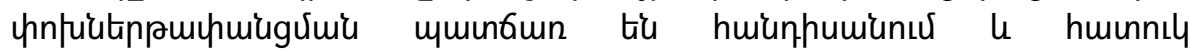

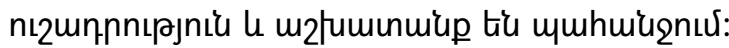




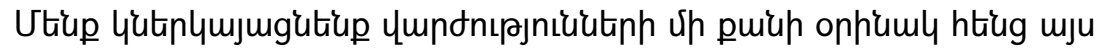

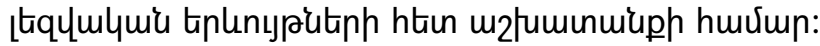

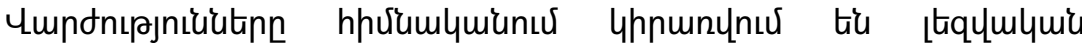

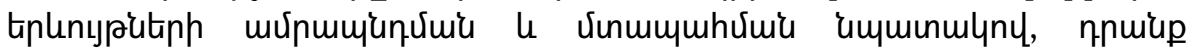

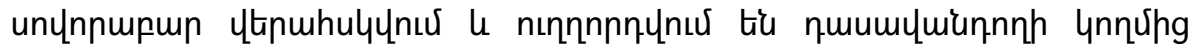

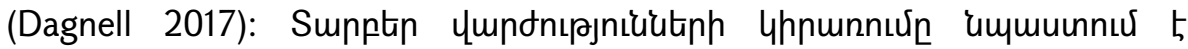

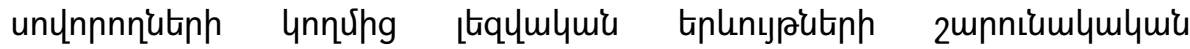

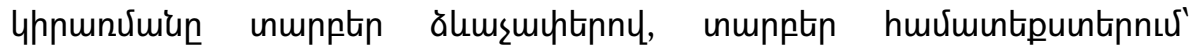

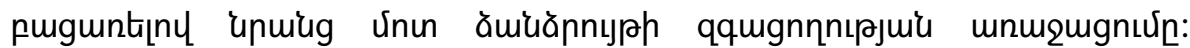

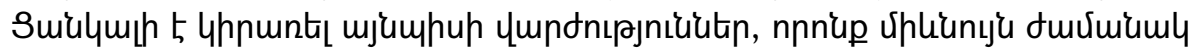

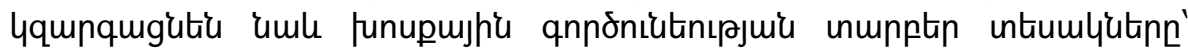

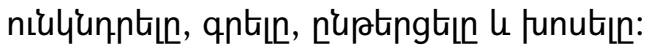

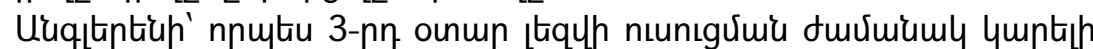

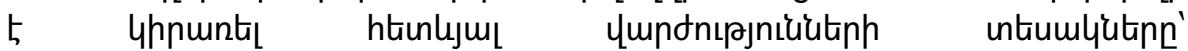

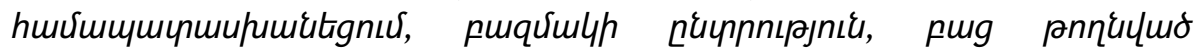

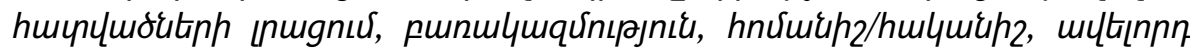

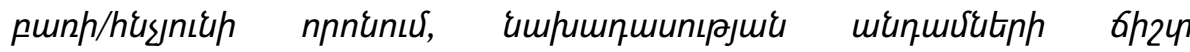

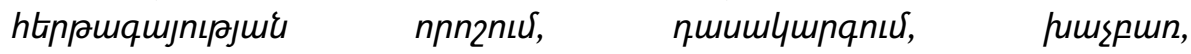

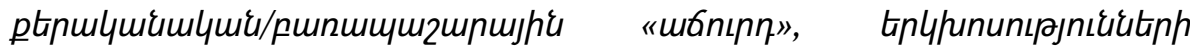

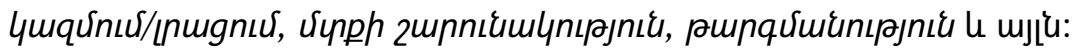

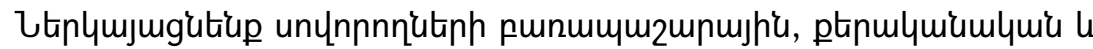

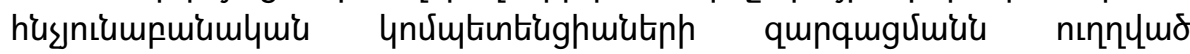

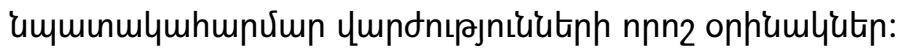

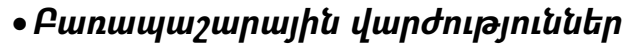

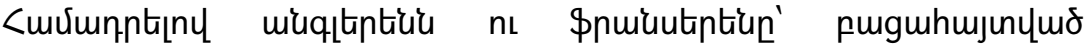

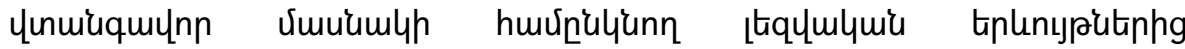

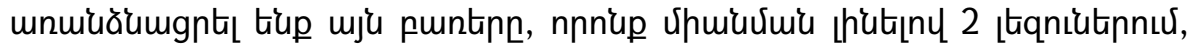

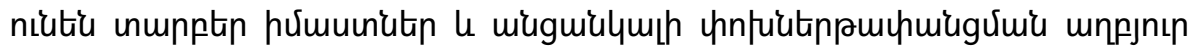

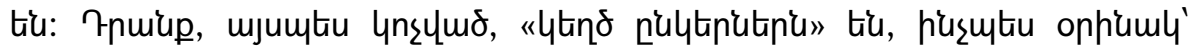
míqutintíu - eventually (h

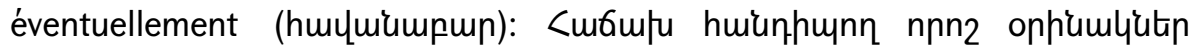
utiplumuggumo tiu Unjnımul 1-nuर:

\begin{tabular}{|c|c|c|c|c|}
\hline $\begin{array}{l}\text { Uuqititiu } \\
\text { punn }\end{array}$ & & $\begin{array}{l}\text { Spuizutintu } \\
\text { huviuundtipn }\end{array}$ & & 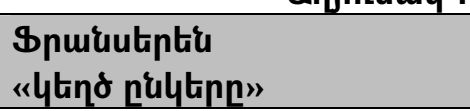 \\
\hline $\begin{array}{l}\text { eventually } \\
\text { (h ytungn) }\end{array}$ & $=$ & finalement & $\neq$ & $\begin{array}{l}\text { éventuellement } \\
\text { (=m'uqtintiu' possibly - } \\
\text { huчu'umpun) }\end{array}$ \\
\hline habit & $=$ & habitude & $\neq$ & habit \\
\hline
\end{tabular}




\begin{tabular}{|c|c|c|c|c|}
\hline (unЦnnnıpjnı¿u) & & & & $\begin{array}{l}\text { (=miuqlaptiu' dress, clothes - } \\
\text { huqniuun) }\end{array}$ \\
\hline 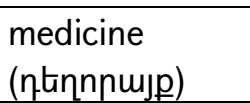 & $=$ & médicament & $\neq$ & $\begin{array}{l}\text { médecin (=miqqutintí' } \\
\text { doctor - pdh24) }\end{array}$ \\
\hline $\begin{array}{l}\text { photograph } \\
\text { ([nıumulyun) }\end{array}$ & $=$ & photographie & $\neq$ & 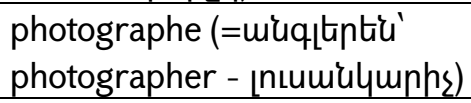 \\
\hline $\begin{array}{l}\text { phrase } \\
\text { (ununuhuj- } \\
\text { innıpjnıu) }\end{array}$ & $=$ & expression & $\neq$ & 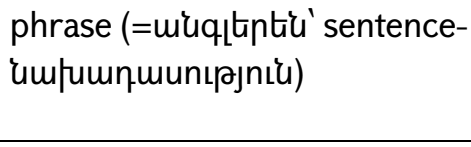 \\
\hline $\begin{array}{l}\text { sensible } \\
\text { (ןut_uúphun) }\end{array}$ & $=$ & raisonnable & $\neq$ & $\begin{array}{l}\text { sensible (= '=uquttintiu' sensitive } \\
\text { - qqujnıu ) }\end{array}$ \\
\hline $\begin{array}{l}\text { sympathetic } \\
\text { (4umblygnn) }\end{array}$ & $=$ & compatissant & $\neq$ & 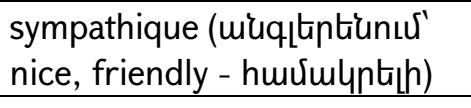 \\
\hline
\end{tabular}

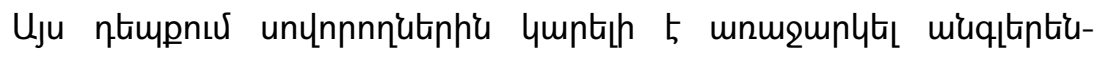

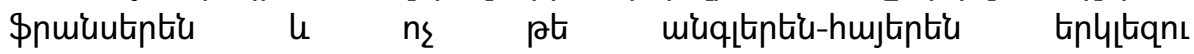

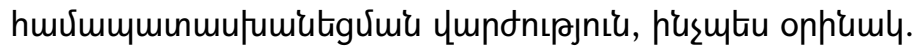

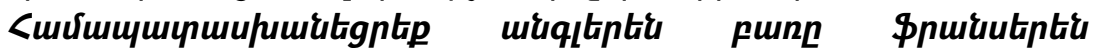

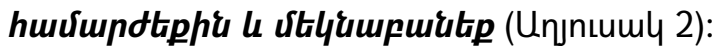

\begin{tabular}{|c|c|c|c|}
\hline & & & Unjnıumly2 \\
\hline & $\begin{array}{l}\text { ENGLISH WORD } \\
\text { (Uし৭LEৎヒし คUกC) }\end{array}$ & & 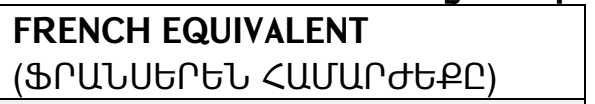 \\
\hline 1. & eventually & a. & photographie \\
\hline 2. & dress & $\mathrm{b}$. & expression \\
\hline 3. & habit & c. & médecin \\
\hline 4. & possibly & d. & phrase \\
\hline 5. & medicine & e. & raisonnable \\
\hline 6. & doctor & f. & habitude \\
\hline 7. & photograph & g. & éventuellement \\
\hline 8. & photographer & $\mathrm{h}$. & finalement \\
\hline 9. & phrase & i. & médicament \\
\hline 10. & sentence & j. & compatissant \\
\hline 11. & sensible & $\mathrm{k}$. & habit \\
\hline 12. & sensitive & I. & photographe \\
\hline 13. & sympathetic & $\mathrm{m}$. & sensible \\
\hline
\end{tabular}




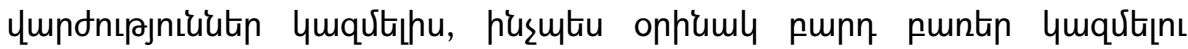

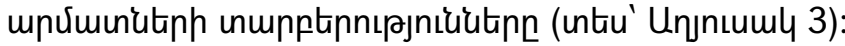

Unjniuml 3

\begin{tabular}{|c|c|c|c|c|}
\hline $\begin{array}{l}\text { Spuiuutnti } \\
\text { punn }\end{array}$ & & $\begin{array}{l}\text { U\{qutiptu } \\
\text { huvuundtpp }\end{array}$ & & Unwidớumhuunlnıpjnitun \\
\hline $\begin{array}{l}\text { petit-enfant }(\mathrm{m}) \\
(p n n)\end{array}$ & $=$ & grandchild & * & 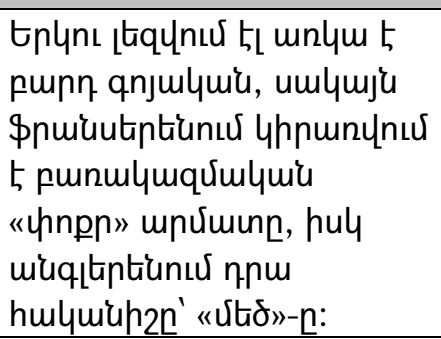 \\
\hline $\begin{array}{l}\text { belle-mère (f) } \\
\text { (ultuunın, } \\
\text { qnpuius) }\end{array}$ & $=$ & mother-in-law & * & 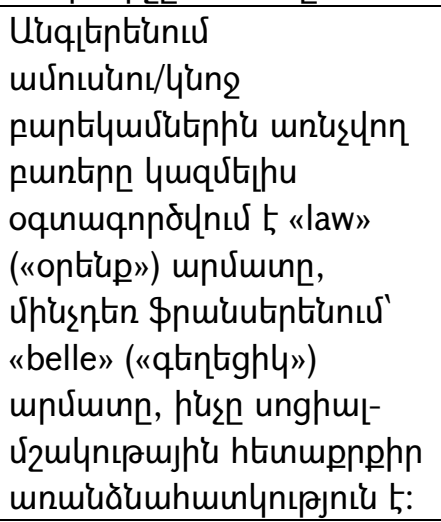 \\
\hline $\begin{array}{l}\text { belle-fille (f) } \\
\text { (hunu, funnp } \\
\text { mnghl) }\end{array}$ & $\approx$ & $\begin{array}{l}\text { daughter-in-law } \\
\text { (hunu), } \\
\text { stepdaughter } \\
\text { (łunnp wngh4) }\end{array}$ & * & 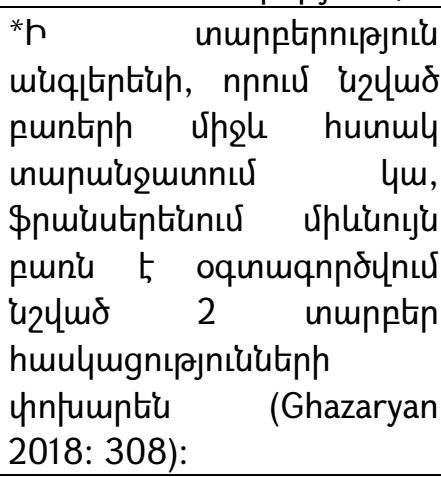 \\
\hline
\end{tabular}

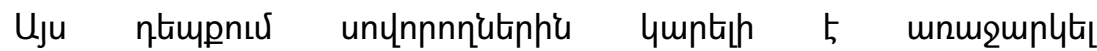

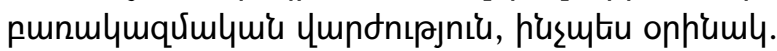

Snyud punting luqútp unn punn puntp' child, in, nice, mother, law, daughter, grand, law, in, little...

- คtpnuluibulyuir yundnıpjnilitin 


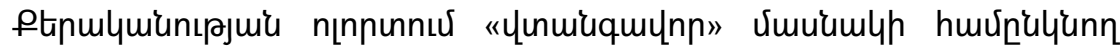

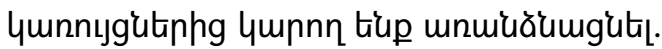

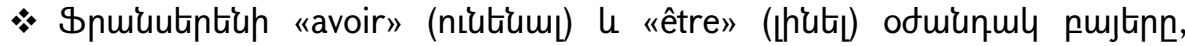

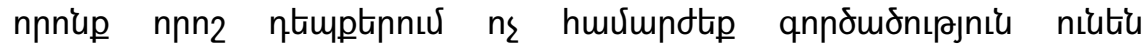
u'uqlaptiunus:

Ophimu'

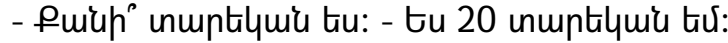

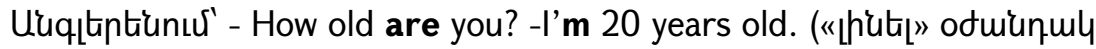
pujny, husułtu hujtintiunux)

Spuiuutntiunux' - Quel age as - tu? - J'ai 20 ans («nı'utiumu» odmiunuly pujny):

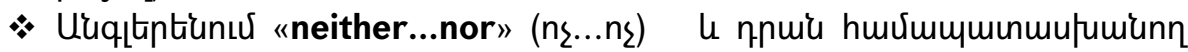

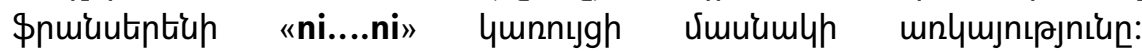

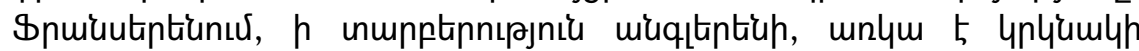

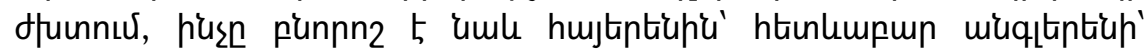

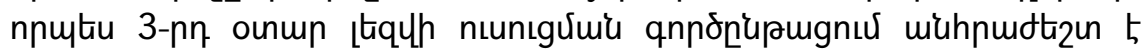

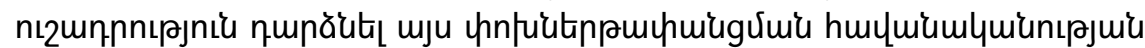

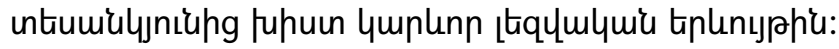

Ophriml'

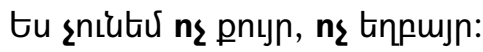

Uuqutintiunud' I have neither sister nor brother.

Spuiuutintiuntu' Je n'ai ni frère, ni sœur.

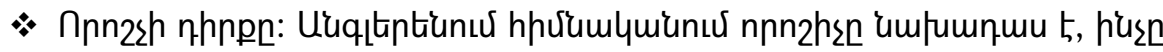

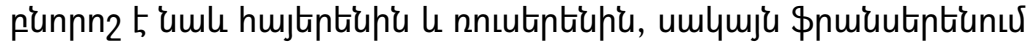

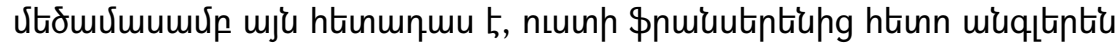

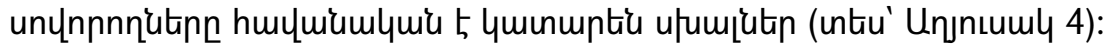

\begin{tabular}{|c|c|c|c|c|}
\hline $\begin{array}{l}\text { Spuirutintí } \\
\text { punn }\end{array}$ & & $\begin{array}{l}\text { Uuqutentu } \\
\text { huviundten }\end{array}$ & & 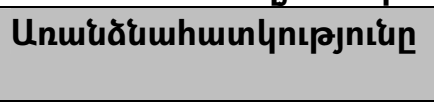 \\
\hline $\begin{array}{l}\text { famille }(f) \\
\text { nucléaire }\end{array}$ & $=$ & nuclear family & * & 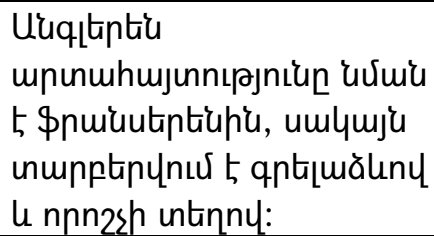 \\
\hline
\end{tabular}

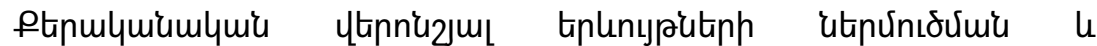

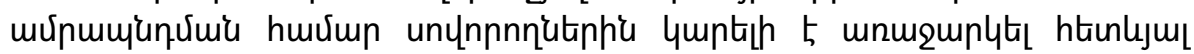




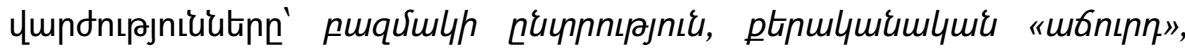

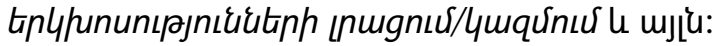

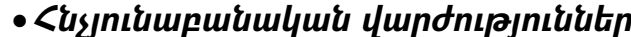

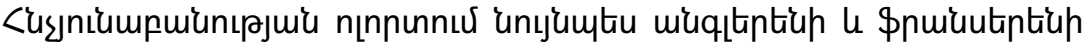

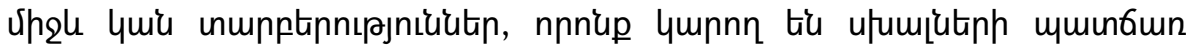

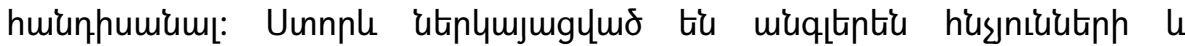

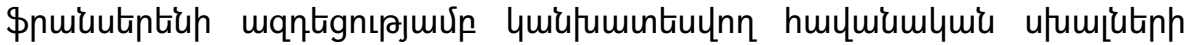
nnn2 oph'umly'utn (untu' Unjnıuml 5):

Unjniumly 5

\begin{tabular}{|c|c|}
\hline UAquttptí husyniun & 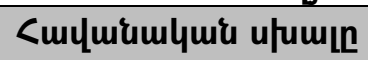 \\
\hline $\begin{array}{l}\text { /ov/ } \\
\text { "hope" }\end{array}$ & $\begin{array}{l}\text { /o/ } \\
\text { "hop" }\end{array}$ \\
\hline $\begin{array}{l}\text { /O/ } \\
\text { "thick" }\end{array}$ & $\begin{array}{l}\text { /t/ } \\
\text { "tick" }\end{array}$ \\
\hline $\begin{array}{l}\text { /ð/ } \\
\text { "then" }\end{array}$ & $\begin{array}{l}\text { /d/ } \\
\text { "den" }\end{array}$ \\
\hline $\begin{array}{l}/ \mathrm{t} / \\
\text { "chin" }\end{array}$ & $\begin{array}{l}/ S / \\
\text { "shin" }\end{array}$ \\
\hline
\end{tabular}

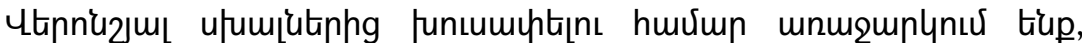

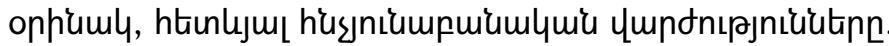

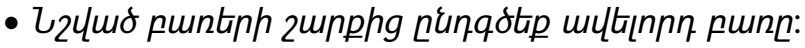

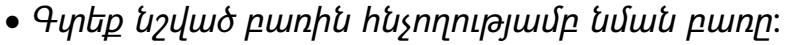

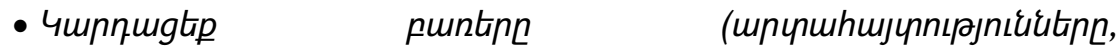

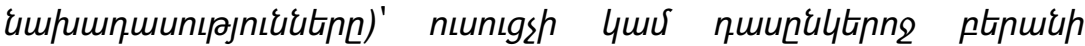
zundnusktinhe htiuntitinu:

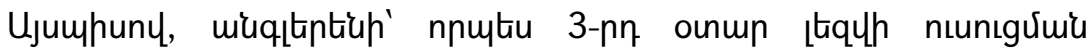

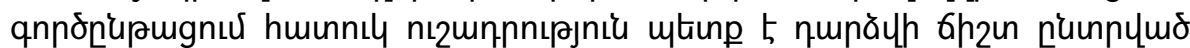

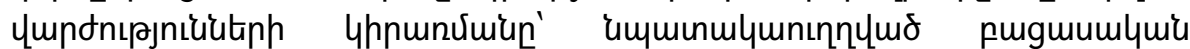

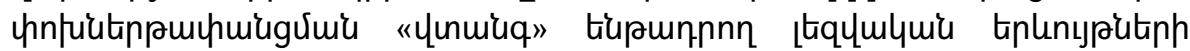

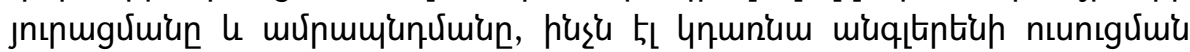

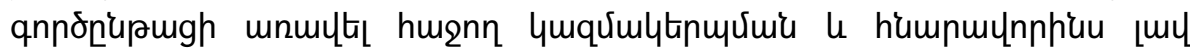
unnjniuputinh mumhnuरumiu qnuumumiu: 


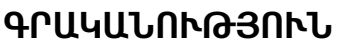

1. Щепилова А. Теория и методика обучения фрранцузскому языку как второму иностранному, М., Владос, 2005.

2. Dagnell, R. (2017). English Language Teaching Materials. Task, Activity, Exercise? Available at:

https://blogs.brighton.ac.uk/tlm25dagnell/2017/05/04/task-activityexercise/\#_ENREF_11 (Accessed 15 March 2021).

3. Ghazaryan, S. (2018) Vocabulary Clustering in the Process of Teaching English After French In Higher Educational Institutions of the Republic of Armenia. Bulletin of Yerevan Brusov State University of Languages and Social Sciences. 2(43). p. 296-312.

4. Jessner, U., Cenoz, J. (2007) Teaching English as a Third Language. Available at:

https://www.researchgate.net/publication/226284939_Teaching_English_a s_a_Third_Language (Accessed 10 March 2021).

\section{REFERENCES}

Shhepilova A. Teorija i metodika obuchenija francuzskomu jazyku kak vtoromu inostrannomu. Vlados. - M.: 2005.

\section{СИРАНУШ КАЗАРЯН - ИСПОЛЬЗОВАНИЕ УПРАЖНЕНИЙ В ПРОЦЕССЕ ОБУЧЕНИЯ АНГЛИЙСКОМУ КАК ТРЕТЬЕМУ ИНОСТРАННОМУ ЯЗЫКУ}

Ключевые слова: интерфреренция, сопоставление, частично совпадающие языковые явления, способность распознавать языки, автоматизация, языковые упражнения

В статье рассматриваются проблемы обучения английскому как третьему иностранному языку в высших учебных заведениях Республики Армения. В данном случае родным языком учащихся является армянский, первым иностранным - русский, а вторым - французский. Учитывая тот факт, что второй (французский) и третий (английский) иностранные языки имеют значительное сходство, которое может оказывать как положительное влияние (транспозицию), так и отрицательное (интерференцию), процесс обучения английскому языку должен быть организован с учетом сходств и некоторых особенностей этих языков. Соответственно, использование правильно подобранных упражнений может способствовать более быстрому и эффективному обучению языку. Автор также иллюстрирует некоторые «опасные» языковые явления, 
которые могут способствовать нежелательной интерференции в изучении английского языка после французского. Кроме того, приводятся образцы упражнений, которые можно использовать для развития лексических, грамматических и фонологических компетенций студентов при обучении английскому как третьему иностранному языку.

\section{SIRANUSH GHAZARYAN - USE OF EXERCISES IN TEACHING/LEARNING ENGLISH AS A THIRD FOREIGN LANGUAGE}

Keywords: interference, comparison, partially overlapping language phenomena, language awareness skill, automation, language exercises

The article touches upon the problems of teaching English as a third foreign language in higher educational institutions of the Republic of Armenia. The students' mother tongue, in this case, is Armenian. Russian is the first foreign language and French is the second one. Considering the fact that the students' $2^{\text {nd }}$ (French) and $3^{\text {rd }}$ (English) foreign languages have significant similarities that can cause both positive transfer and negative interference, the teaching/learning process of English should be organized by paying special attention to the similarities and taking into account certain peculiarities. Accordingly, the use of correctly selected exercises can help in organizing the teaching/learning process more quickly and effectively. The author also introduces some "dangerous" language phenomena that may bring about undesirable interference in learning English after French. In addition, a number of exercise samples are provided that might be used to develop the students' lexical, grammatical and phonological competences in teaching/learning English as a third foreign language.

Utplumugyltis 5 ' 22.04.2021

9pulunultil $h^{\prime} \quad 20.04 .2021$ 